\title{
Quantum dot-based diabetic foot mapping for diagnosing osteomyelitis and Charcot neuroarthropathy
}

\author{
Farzaneh Yousefi ${ }^{a}$, Iraj Nabipour ${ }^{a}$, Mohammadreza Kalantarhormozi ${ }^{a}$, Tahereh Assadi ${ }^{\text {a }}$, Alireza Raeisi ${ }^{a}$, \\ Majid Assadi ${ }^{\text {b,* }}$ \\ a Department of Endocrinology, The Persian Gulf Tropical Medicine Research Center, Bushehr University of Medical Sciences, Bushehr, Iran \\ ${ }^{\mathrm{b}}$ The Persian Gulf Nuclear Medicine Research Center, Bushehr University of Medical Sciences, Bushehr, Iran
}

\section{A R T I C L E I N F O}

\section{Article history:}

Received 7 January 2015

Accepted 7 March 2015

\begin{abstract}
A B S T R A C T
The location of osteomyelitis is very important in Charcot neuroarthropathy $(\mathrm{CN})$, especially when a physician is considering amputation of the affected extremity.

In diabetic $\mathrm{CN}$, the presence of osteomyelitis is likely. Thus, to identify the infected tissue that needs to be removed, the specific area of infection must be correctly identified. Both $\mathrm{CN}$ and osteomyelitis have high mortality rates, but osteomyelitis is more life threatening and needs aggressive treatment.

We propose a QD-based method for distinguishing CN with sterile inflammation from osteomyelitis that does not require multiple and frequent imaging modalities. The method utilizes two different colored QDs (i.e., red and green). The red QD is attached to a UBI, an antimicrobial peptide, which attaches to bacteria, enabling their detection. The green QD is attached to MDP, which accumulates in areas of inflammation. When these QDs are injected intravenously at the same time, the red QD-UBI accumulates in infected areas and attaches to bacteria, and the green QD-MDP accumulates both in areas with sterile inflammation and infected areas.

The accumulation of only green QDs in the suspect extremity signifies a sterile inflammation process $(\mathrm{CN})$. However, the accumulation of both the red and green QDs signify infectious and inflammation processes (i.e., osteomyelitis or a soft tissue infection, depending on the location). In the latter case, the treatment needs to be more intensive, with even amputation considered.
\end{abstract}

(c) 2015 Elsevier Ltd. All rights reserved.

\section{Introduction}

Diabetes is one of the most common diseases in the world, and its prevalence is increasing rapidly. According to a report by the American Diabetes Association, the number of diabetic patients in the U.S. increased from 25.8 million in 2010 to 29.1 in 2012 [1]. Diabetes is not a curable disease, but it can be managed to prevent serious consequences, such as neuropathy, retinopathy, diabetic nephropathy, gastric paresis, cardiovascular disease, hypertension, and infections [2].

\section{Osteomyelitis}

Osteomyelitis of the foot in diabetic patients is largely a consequence of several diabetes-related complications, especially

* Corresponding author at: The Persian Gulf Nuclear Medicine Research Center, Bushehr University of Medical Sciences, Bushehr 3631, Iran. Tel.: +98 771 2580169; fax: +987712541828.

E-mail addresses: assadipoya@yahoo.com, asadi@bpums.ac.ir (M. Assadi). neuropathy, and, to a lesser degree, vasculopathy and defects in immunity and wound healing [3]. In osteomyelitis, the infarcted part of the foot (usually the forefoot or a toe) is dull red and diffusely swollen and warm. It may also be discharging pus or fragments of bone. Systemic signs of disease, such as fever and malaise, are unusual with foot infections, including osteomyelitis [4-10]. A clinical examination may lead to a suspicion of osteomyelitis, but further evaluation is needed to confirm the disease. If bone can be felt with the tip of a sterile metal probe inserted in the wound (probe-to-bone test), then bone infection (defined histologically) is likely. The probe-to-bone test has a sensitivity of only $66 \%$, but it is relatively specific (84\%) and has a negative predictive value of $62 \%$. Laboratory findings, such as leukocytosis, and tests of ESR and CRP, which are increased in osteomyelitis, are useful in the diagnosis. A study showed that ESR higher than $70 \mathrm{~mm} / \mathrm{h}$ indicated bone infection, with $100 \%$ specificity and $50 \%$ sensitivity [5]. Imaging modalities are also helpful in the diagnosis of osteomyelitis. Plain X-rays have poor sensitivity in the early stages of the disease [6]. In contrast, ${ }^{99 \mathrm{~m}} \mathrm{Tc}$-diphosphonate can demonstrate abnormal uptake as long as 2 weeks before abnormalities are seen on plain radiographs 
[7]. As ${ }^{111}$ In-labeled leukocytes are not incorporated into areas of active bone turnover, the accumulation of labeled leukocytes is relatively specific for infection, especially in cases where other osseous abnormalities are present [7]. Tc-UBI, a Tc-labeled cationic antimicrobial peptide derived from ubiquicidin, binds strongly to microorganisms [8]. Akhtar et al. showed that the sensitivity, specificity, and overall diagnostic accuracy of TC-UBI 29-41 in infection localization were $100 \%, 80 \%$, and $94 \%$, respectively. The positive and negative predictive values of TC-UBI 29-41 were $92.9 \%$ and $100 \%$, respectively [9]. In previous study, the accuracy of a $99 \mathrm{~m}$ Tc-UBI scan in the diagnosis of osteomyelitis was very high [9].

MRI has an important role in the diagnosis of osteomyelitis. Marrow edema associated with inflammation is responsible for the characteristic changes seen in MRI of osteomyelitis. The diagnostic sensitivity of MRI for osteomyelitis has generally been reported to be $90-100 \%$. Its specificity is somewhat limited due to the difficulty in MRI distinguishing osteomyelitis from other causes of marrow edema, including acute neuropathic osteoarthropathy [10-12].

A bone biopsy in diabetic patients with suspected osteomyelitis can be done. However, it is an expensive procedure, requires experience and technical skills, and takes several days to process [13]. It may also yield false negative results, either because of patchy infectious involvement or because of previous antibiotic therapy [10].

Osteomyelitis is a difficult infection to treat, largely because of limited blood flow in bone. As relapsing or repeated infections are common in diabetic foot osteomyelitis, surgical resection of the infected bone is often necessary $[3,14]$.

\section{Diabetic Charcot neuroarthropathy}

Charcot neuroarthropathy $(\mathrm{CN})$ is a condition affecting the bones, joints, and soft tissues of the foot and ankle, and it is characterized by inflammation in the earliest phases [15]. The clinical presentation and imaging findings of $\mathrm{CN}$ are challenging, as they are very similar to those of osteomyelitis. Both $\mathrm{CN}$ and osteomyelitis can coexist in an extremity, making the diagnosis even harder [16-19].

The sensitivity and specificity $(<50 \%)$ of plain radiography in detecting early-stage $\mathrm{CN}$ are low [20]. Technetium-99m methylene diphosphonate $99 \mathrm{~m}$ (TC-MDP) is positive in all three stages of $\mathrm{CN}$ and reflects the increased turnover of bone $[20,21]$. 99m TC and 111In-WBC do not accumulate at sites of new bone formation without infection [21]. MRI is the most sensitive modality in detecting early changes in $\mathrm{CN}$, with $90-100 \%$ sensitivity and 40-100\% specificity [21-25].

The treatment aim in $\mathrm{CN}$ is to arrest the acute process to prevent the development of permanent deformity and to relieve pain, mostly by conservative treatment [26-28].

\section{Quantum dots (QDs)}

Quantum dots (QDs), tiny light-emitting particles at the nanometer scale, are emerging as a new class of fluorescent probe for in vivo bimolecular and cellular imaging [29]. QDs are extremely uniform, possess a high surface to volume ratio, and are endowed with intrinsic fluorescent properties, including very bright intensity and photostability. Moreover, the intermittent fluorescence emission (optical blinking) and electron dense nature of QD nanoparticles allow for easy identification of individual nanoparticles in cell preparations [30,31]. In vitro applications of QDs are bimolecular tracking in cells, cellular imaging, and tissue staining. In vivo applications of QDs are biodistribution [32], vascular imaging [33], QD tracking [34], and tumor imaging [35].

With the development of biomarkers in cell biology, the tracking of some specific cells (such as cancer cells) becomes possible. Gac et al. have successfully detected apoptotic cells by conjugating QDs with biotinylated Annexin V, which enables the functionalized QDs to bind to phosphatidylserine (PS) moieties present on the membrane of apoptotic cells but not on healthy or necrotic cells [36]. Liu et al. reported the development of Gd-doped ZnO QDs with enhanced yellow fluorescence, and these QDs can be used as nanoprobes for quick cell detection with very low toxicity [37]. In 2007, Bagalkot et al. reported a more complex QDsaptamer- (Apt-) doxorubicin (Dox) conjugate system [QDApt(Dox)] to endow QDs with the capability of targeting, imaging, therapy, and sensing the prostate cancer cells that express the prostate-specific membrane antigen (PSMA) protein [38].

In addition to their usage as nanoprobes and labels for in vitro imaging, QDs have also been widely used as in vivo imaging agents. Cancer-specific antibody, coupled to near-IR QDs with polymer coatings is the most popular QDs agent for tumor targeted imaging [39]. One study used nude mice for in vivo imaging after near-IR QD800-labeled BcaCD885 cells (BcaCD885/QD800) being implanted. Fluorescence signals of QDs accumulated in the tumor could be detected after 16 days of incubation at certain concentrations. It suggested that, compared with CT and MRI, QD800-based imaging could efficiently increase the sensitivity of early diagnosis of cancer cells [40].

\section{Hypothesis: QD-based method for distinguishing $\mathrm{CN}$ and osteomyelitis}

Due to the similar clinical presentation of $\mathrm{CN}$ and osteomyelitis and their similar imaging findings, the diseases are not easily distinguishable, but they require different management and treatment.

The location of osteomyelitis is very important in $\mathrm{CN}$, especially when a physician is considering amputation of the affected extremity.

In diabetic $\mathrm{CN}$, the presence of osteomyelitis is likely. Thus, to identify the infected tissue that needs to be removed, the specific area of infection must be correctly identified. Both $\mathrm{CN}$ and osteomyelitis have high mortality rates, but osteomyelitis is more life threatening and needs aggressive treatment.

We propose a QD-based method for distinguishing $\mathrm{CN}$ with sterile inflammation from osteomyelitis that does not require multiple and frequent imaging modalities (Fig. 1). The method utilizes two different colored QDs (i.e., red and green). The red QD is attached to a UBI, an antimicrobial peptide, which attaches to bacteria, enabling their detection. The green QD is attached to MDP, which accumulates in areas of inflammation. When these QDs are injected intravenously at the same time, the red QD-UBI accumulates in infected areas and attaches to bacteria, and the green QD-MDP accumulates both in areas with sterile inflammation and infected areas.

The accumulation of only green QDs in the suspect extremity signifies a sterile inflammation process $(\mathrm{CN})$. However, the accumulation of both the red and green QDs signify infectious and inflammation processes (i.e., osteomyelitis or a soft tissue infection, depending on the location). In the latter case, the treatment needs to be more intensive, with even amputation considered. 

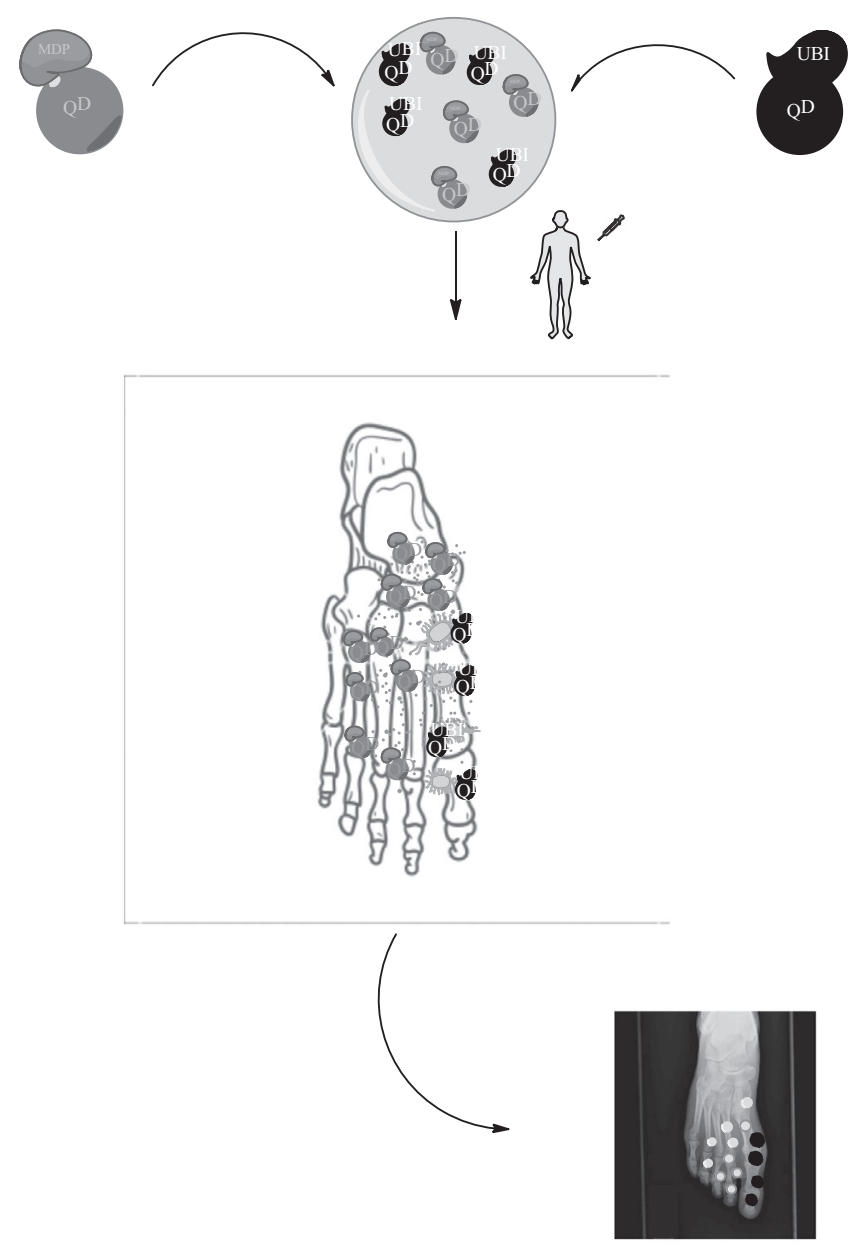

Fig. 1. QD-based method for distinguishing $\mathrm{CN}$ and osteomyelitis.

\section{Disclosure statement}

The authors have nothing to disclose.

\section{References}

[1] American Diabetes Association. Data from the National Diabetes Statistics Report, 2014. http://www.diabetes.org/diabetesbasics/statistics/\#sthash. SSa67Oia.dpuf (released June 10, 2014).

[2] Fauci, Branwalld, Kasper, et al. Harrison's principles of internal medicine, McGraw Hill; 2012. ISBN-13:9780071748896.

[3] Lipsky B. Osteomyelitis of the foot in diabetic patients. CID 1997;25:1318-26.

[4] Sapico FL, Canawati HN, Witte JL, Montgomerie JZ, Wagner Jr FW, Bessman AN. Quantitative aerobic and anaerobic bacteriology of infected diabetic feet. J Clin Microbiol 1980;12:413-20.

[5] Eneroth M, Larsson J, Apelqvist J. Deep foot infections in patients with diabetes and foot ulcer: an entity with different characteristics, treatments, and prognosis. J Diabetes Complications 1999;13:254-63.

[6] Eckman MH, Greenfield S, Mackey WC, et al. Foot infections in diabetic patients. Decision and cost-effectiveness analyses. JAMA 1995;273:712-20.

[7] Newman LG, Waller J, Palestro CJ, et al. Unsuspected osteomyelitis in diabetic foot ulcers: diagnosing and monitoring by leukocyte scanning with indium In ${ }^{111}$ oxyquinolone. JAMA 1991;266:1246-51.

[8] Lupetti A, Welling MM, Paulwels EK, Nibbering PH. Radiolabeled antimicrobial peptides for infection detection. Lancet Infect Dis 2003;3:223-9.
[9] Akhtar MS, Qaisar A, Irfanullah J, et al. Antimicrobial peptide TC-Ubiquicidin 29-41 as human infection-imaging agents: clinical trial. J Nucl Med 2005;46:567-73.

[10] Jeffcoate W, Lipsky B. Controversies in diagnosis and managing osteomyelitis of the foot in diabetes. CID 2004;39(2):115-22.

[11] Croll SD, Nicholas GG, Osborne MA, Wasser TE, Jones S. Role of magnetic resonance imaging in the diagnosis of osteomyelitis in diabetic foot infections. J Vasc Surg 1996;24:266-70.

[12] Craig JG, Amin MB, Wu K, et al. Osteomyelitis of the diabetic foot: MR imaging - pathologic correlation. Radiology 1997;203:849-55.

[13] Lipsky BA. Osteomyelitis of the foot in diabetic patients. Clin Infect Dis 1997;25:1318-26.

[14] Cierny G, Mader JT, Pennick JJ. A clinical staging system of adult osteomyelitis. Contemp Orthopaedics 1985;10:17-37.

[15] Rogers L, Frykberg R, Armstrong D, et al. The Charcot foot in diabetes. Diabetes Care 2011;34:2123-9.

[16] Armstrong DG, Todd WF, Lavery LA, Harkless LB, Bushman TR. The natural history of acute Charcot's arthropathy in a diabetic foot speciality clinic. Diabetic Med 1997;14:357-63.

[17] Sella EJ, Barrette C. Staging of Charcot neuroarthropathy along the medial column of the foot in the diabetic patient. J Foot Ankle Surg 1999;38:34-40.

[18] Rajbhandari SM, Jenkins RC, Davies C, Tesfaye S. Charcot neuroarthropathy in diabetes mellitus. Diabetologia 2002;45:1085-96.

[19] Caputo GM, Ulbrecht J, Cavanagh PR, Juliano P. The Charcot foot in diabetes: six key points. Am Fam Physician 1998;57:2705-10.

[20] Rajbhandri SM, Jenkins RC, Davies C, Tesfaye S. Charcot neuroarthropathy in diabetes mellitus. Diabetologia 2002;45:1085-96.

[21] Sanverdi S, Ergen F, Oznur A. Current challenges in imaging of the diabetic foot. Diabetic Foot Ankle 2012;3:1-17.

[22] Schweitzer ME, Daffner RH, Weissman BN, Bennett DL, Blebea JA, Jacobson A, et al. ACR appropriateness criteria on suspected osteomyelitis in patients with diabetes mellitus. J Am Coll Radiol 2008;5:881-6.

[23] Roug IK, Pierre-Jerome C. MRI spectrum of bone changes in the diabetic foot. Eur J Radiol 2012;81:1625-9.

[24] Palestro C, Love C. Nuclear medicine and diabetic foot infections. Semin Nucl Med 2009;39:53-65.

[25] Toledano TR, Fatone EA, Weis A, Cotten A, Beltran J. MRI evaluation of bone marrow changes in the diabetic foot: a practical approach. Semin Musculoskelet Radiol 2011;15:257-68.

[26] Frykberg RG, Mendeszoon E. Management of the diabetic Charcot foot. Diabetes Metab Res Rev 2000;16(Suppl. 1):S59-65.

[27] Henderson VE. Joint affection in tabes dorsalis. J Pathol 1905;10:211.

[28] Assadi M, Vahdat K, Nabipour I, et al. Diagnostic value of TC-ubiquicidin scintigraphy for osteomyelitis and comparisons with TC-methylene diphosphonate scintigraphy and Magnetic resonance imaging. Nucl Med Commun 2011.

[29] Gao X, Yang L, Petros J, et al. In vivo molecular and cellular imaging with quantum dots. Curr Opin Biotechnol 2005;16(1):63-72.

[30] Vu TQ et al. Peptide conjugated quantum dots activate neuronal receptors and initiate downstream signaling of neurite outgrowth. Nano Lett 2005;5:603-7.

[31] Lidke DS et al. Quantum dot ligands provide new insights into erbB/HER receptor-mediated signal transduction. Nat Biotechnol 2004;22:198-203.

[32] Soltesz E, Kim S, Laurence R, et al. Intraoperative sentinel lymph node mapping of the lung using near-infrared fluorescent quantum dots. Ann Thorac Surg 2005;79:269-77.

[33] Kim S, Lim YT, Soltesz EG, et al. Near-infrared fluorescent Type II quantum dots for sentinel lymph node mapping. Nat Biotechnol 2004;22:93-7.

[34] Gao XH, Cui YY, Levenson RM, et al. In vivo cancer targeting and imaging with semiconductor quantum dots. Nat Biotechnol 2004;22:969-76.

[35] Akerman ME, Chan WCW, Laakkonen P, et al. Nanocrystal targeting in vivo. Proc Natl Acad Sci U S A 2002:99:12617-21.

[36] Gac SL, Vermes I, Berg AVD. Quantum dots based probes conjugated to annexin $\mathrm{V}$ for photostable apoptosis detection and imaging. Nano Lett 2006;6(9): 1863-9.

[37] Liu A, Peng S, Soo JC, Kuang M, Chen P, Duan H. Quantum dots with phenylboronic acid tags for specific labeling of sialic acids on living cells. Anal Chem 2011;83(3):1124-30.

[38] Bagalkot V, Zhang LF, Levy-Nissenbaum E, et al. Quantum dot-aptamer conjugates for synchronous cancer imaging, therapy, and sensing of drug delivery based on bifluorescence resonance energy transfer. Nano Letters 2007;7(10):3065-70.

[39] Li L, Daou TJ, Texier I, Chi TTK, Liem NQ, Reiss P. Highly luminescent cuins $2 /$ ZnS core-shell nanocrystals: cadmium-free quantum dots for in vivo imaging. Chem Mater 2009;21(12):2422-9.

[40] Yang K, Cao YA, Shi C, et al. Quantum dot-based visual in vivo imaging for oral squamous cell carcinoma in mice. Oral Oncol 2010;46(12):864-8. 